\title{
Comprehensive efficiency analysis of organic light-emitting diodes featuring emitter orientation and triplet-to-singlet up- conversion
}

Cite as: Appl. Phys. Lett. 103, 093303 (2013); https://doi.org/10.1063/1.4819388

Submitted: 12 June 2013 . Accepted: 11 August 2013 . Published Online: 27 August 2013

Tobias D. Schmidt, Daniel S. Setz, Michael Flämmich, Jörg Frischeisen, Dirk Michaelis, Christian Mayr, Andreas F. Rausch, Thomas Wehlus, Bert J. Scholz, Thilo C. G. Reusch, Norbert Danz, and Wolfgang Brütting

\section{ARTICLES YOU MAY BE INTERESTED IN}

Evidence for non-isotropic emitter orientation in a red phosphorescent organic light-emitting diode and its implications for determining the emitter's radiative quantum efficiency Applied Physics Letters 99, 163302 (2011); https://doi.org/10.1063/1.3653475

Determination of molecular dipole orientation in doped fluorescent organic thin films by photoluminescence measurements

Applied Physics Letters 96, 073302 (2010); https://doi.org/10.1063/1.3309705

Extracting the emitter orientation in organic light-emitting diodes from external quantum efficiency measurements

Applied Physics Letters 105, 043302 (2014); https://doi.org/10.1063/1.4891680

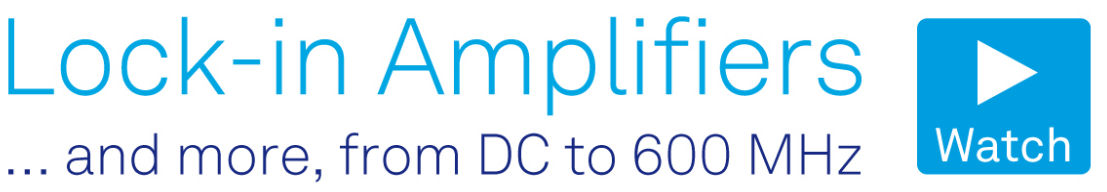




\title{
Comprehensive efficiency analysis of organic light-emitting diodes featuring emitter orientation and triplet-to-singlet up-conversion
}

\author{
Tobias D. Schmidt, ${ }^{1, a)}$ Daniel S. Setz, ${ }^{2}$ Michael Flämmich, ${ }^{3}$ Jörg Frischeisen, ${ }^{1}$ \\ Dirk Michaelis, ${ }^{3}$ Christian Mayr, ${ }^{1}$ Andreas F. Rausch, ${ }^{2}$ Thomas Wehlus, ${ }^{2}$ Bert J. Scholz, ${ }^{1}$ \\ Thilo C. G. Reusch, ${ }^{2}$ Norbert Danz, ${ }^{3}$ and Wolfgang Brütting ${ }^{1, b)}$ \\ ${ }^{1}$ Institute of Physics, University of Augsburg, 86135 Augsburg, Germany \\ ${ }^{2}$ OSRAM Opto Semiconductors GmbH, Leibnizstrasse 4, 93055 Regensburg, Germany \\ ${ }^{3}$ Fraunhofer Institute for Applied Optics and Precision Engineering, 07745 Jena, Germany
}

(Received 12 June 2013; accepted 11 August 2013; published online 27 August 2013)

\begin{abstract}
We present a method to achieve a consistent, comprehensive efficiency analysis of fluorescent organic light-emitting diodes (OLEDs) showing non-isotropic emitter orientation and triplet-tosinglet up-conversion. Combining photoluminescence lifetime and external quantum efficiency measurements on OLEDs with varying cavity length allows for an independent determination of the radiative emitter efficiency under optical as well as electrical excitation. The difference clearly shows a significant enhancement of the singlet exciton fraction to more than $25 \%$ under electrical operation. Furthermore, the presented method does not require detailed information about the emitting system and is generally applicable for a comprehensive efficiency analysis of bottom-emitting OLEDs. (C) 2013 AIP Publishing LLC. [http://dx.doi.org/10.1063/1.4819388]
\end{abstract}

In recent years, organic light-emitting diodes (OLEDs) entered the commercial sector, especially for mobile display applications. For lighting applications, first products have been launched with an emphasis on design and high-end luminaires. ${ }^{1}$ However, there is still much room for improvement in terms of efficiency and long-term stability during electrical operation. One important shortcoming is the lack of stable phosphorescent emitting systems in the deep-blue color range. ${ }^{2}$ Hence, alternative approaches, such as fluorescent emitters using triplet-to-singlet up-conversion, may serve as promising alternatives. Previous publications have demonstrated that it is possible to enhance the fluorescent radiative exciton fraction using effects, such as triplettriplet-annihilation (TTA) ${ }^{3-6}$ and thermally activated delayed fluorescence (TADF). ${ }^{7-9}$ However, the relevant singlet exciton fraction has only been extracted indirectly so far, mostly from an enhancement of the internal quantum efficiency (IQE) beyond the value of $25 \%$ compatible with the spin statistical limit.

What is actually measured is the external quantum efficiency (EQE) of an OLED, which is given by ${ }^{10}$

$$
\mathrm{EQE}=\gamma \times \eta_{\mathrm{r}} \times q_{\mathrm{eff}}(q) \times \eta_{\text {out }},
$$

where $\gamma$ is the charge carrier balance and can be considered as unity if appropriate blocking and conductivity doped transport layers are used in the device. ${ }^{11,12} \eta_{\mathrm{r}}$ represents the fraction of excitons that is allowed to decay radiatively by quantum mechanical selection rules. This factor is unity for phosphorescent emitters, ${ }^{13-15}$ while it is assumed to be $25 \%$ for fluorescent ones. ${ }^{16,17} q_{\text {eff }}$ is the effective radiative quantum efficiency (RQE) of the emitter material, which depends on the intrinsic radiative quantum efficiency ( $q$, in the following denoted as RQE) modified by the Purcell effect, if

\footnotetext{
${ }^{\text {a)} E l e c t r o n i c ~ m a i l: ~ T o b i a s . S c h m i d t @ p h y s i k . u n i-a u g s b u r g . d e ~}$

${ }^{b}$ Electronic mail: Wolfgang.Bruetting@physik.uni-augsburg.de
}

the emitter is embedded in a microcavity-like structure, such as in an OLED. ${ }^{18-20}$ Finally, $\eta_{\text {out }}$ represents the light outcoupling factor, which is mainly determined by the optical environment, i.e., the refractive indices and thicknesses of the layers used in the device, and the orientation of the lightemitting molecules.

An inherent problem with Eq. (1) is that conclusions on the radiative exciton fraction $\eta_{\mathrm{r}}$ and possible enhancements by TTA or TADF from measured EQE values alone are not straight-forward as long as the other factors are not known exactly. In particular, it has been shown ${ }^{21-23}$ that both the radiative rate of the emitting system inside the OLED cavity as well as the light outcoupling factor of the device are affected by non-isotropic emitter orientation. Therefore, we present an efficiency analysis in this paper, which is based on systematic changes of the cavity strength at the emitter position inside the OLED by layer thickness variation. ${ }^{22-24}$ We independently determine the emitter orientation and the radiative emitter quantum efficiency from optical experiments. Together with the assumption of well-balanced charge carriers $(\gamma=1)$, the measured EQE data thus directly yield information about the radiative exciton fraction. The generality of our approach is demonstrated with a blue fluorescent OLED, where an enhancement of the radiative exciton fraction beyond the spin-statistical limit is clearly identified, without knowing details of the chemical structure of the involved materials.

Figure 1(a) schematically illustrates the layer stack of the OLEDs under investigation, comprising conductivity doped transport and appropriate blocking layers. The thickness of the electron transporting layer (ETL) was varied to achieve the required variation of the cavity length of the OLED. It was verified that the charge carrier balance of the devices is independent of the actual transport layer thicknesses; it can hence be safely assumed to be close to unity. The emission layer was prepared by co-evaporation of a matrix material and a fluorescent dye (some wt. \%). For 
(a)

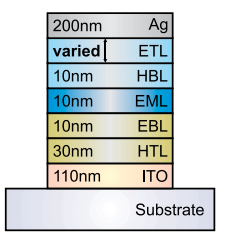

(b)

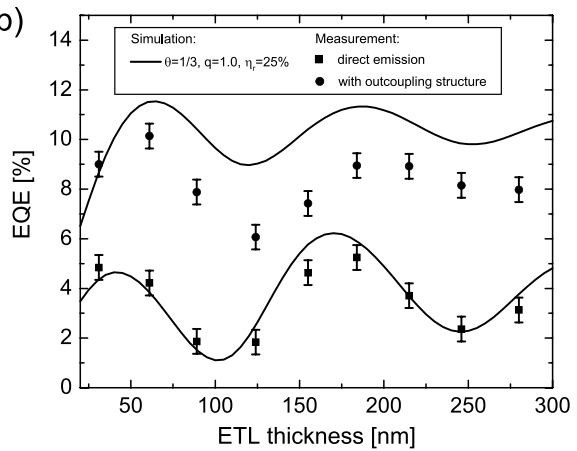

FIG. 1. (a) Stack layout of the OLEDs under investigation. An energy level diagram of the used organic materials is given in the supplementary information. ${ }^{29}$ The ETL thickness was varied to achieve changes of the cavity strength at the emitter's position inside the OLED. (b) Comparison of measured EQE values at a current density of $2 \mathrm{~mA} / \mathrm{cm}^{2}$ (squares: direct emission; dots: with macroextractor) and optical simulations using $\eta_{\mathrm{r}}=25 \%$ and an isotropic emitter orientation (solid lines). The best fit of the direct emission is achieved with an RQE value of unity. The behavior of the extracted substrate modes is simulated with the same values as for the direct emission.

information about the energy levels of each organic material used in the devices, it should be referred to Ref. 29. As mentioned above, chemical structures of the materials are not relevant in the context of our approach, however several examples of blue fluorescent OLEDs with suspected $\mathrm{IQE}>25 \%$ have been published in the literature..$^{3-5,7,8}$ All that is required for optical simulations of the radiative properties (emission lifetime and light outcoupling) are the optical constants and thicknesses of all layers of the OLED stack and the (intrinsic) emitter spectrum. ${ }^{25}$

The analysis of the EQE measurements of the devices with varying ETL thickness for the direct emission and with a macroscopic outcoupling structure (glass hemisphere) that is index-matched to the substrate, giving access to the captured substrate modes, at a current density of $2 \mathrm{~mA} / \mathrm{cm}^{2}$ is shown in Fig. 1(b). Both data sets show the typical oscillations as a function of the emitter/cathode distance. The current density was chosen to avoid current induced quenching processes, such as TTA or triplet polaron quenching. ${ }^{26-28}$ The effect of the current density on the external quantum efficiency was checked for a large current density range and the chosen value for the EQE measurements used in the efficiency analysis is far away from the beginning of significant efficiency roll-off (see supplementary information for details $^{29}$ ). All EQE values presented in this paper have been measured in a calibrated integrating sphere.

First, the experimental results of the direct emission are compared with simulations (for details of the underlying theory we refer to Refs. 22-24) for an isotropic emitter orientation, a radiative exciton fraction $\left(\eta_{\mathrm{r}}\right)$ of $25 \%$ and the $\mathrm{RQE}$ of the emitting system as free parameter. Although, the measured data points are not perfectly following the simulated curve, it is obvious that the RQE would have to be very close to unity to explain the measured behavior of the direct emission (squares in Fig. 1(b)). However, it is apparent that the substrate modes cannot be described in a proper way by this parameter set. Thus, the assumption of an isotropic emitter orientation and/or a radiative exciton fraction of $25 \%$ (classical spin statistics) have to be revised.

One possible explanation for achieving the measured EQE values with RQE values $q<1$ is an orientation of the transition dipole moments of the emitting species parallel to the substrate plane, resulting in an increased light outcoupling factor compared with an isotropic distribution. ${ }^{21-23,30,31}$ Thus, a determination of the anisotropy factor ${ }^{22} \theta$ of the emitting system, defined as the fraction of vertically oriented to the total amount of transition dipole moments, by means of combined angular and polarization dependent photo- and electroluminescence measurements is required. ${ }^{32,33}$ Figure 2(a) demonstrates the measured p-polarized angular dependent emission cross section at a wavelength of $470 \mathrm{~nm}$ for a device with an ETL thickness of $67 \mathrm{~nm}$ (however without cathode). The best fit results in a predominantly horizontal emissive dipole distribution with an anisotropy factor of $\theta=0.2 / 2.2=0.09$, which is in very good agreement with the electrical analysis for an ETL thickness of $91 \mathrm{~nm}$ next to the cavity minimum of the OLED (not shown here). For a detailed description of these two methods and the used equipment, we refer to Frischeisen et $a l^{32}$ and Flämmich et al. ${ }^{33}$ With that information, the modified best fit of the direct emission (shown in Fig. 2(b)) yields an RQE of

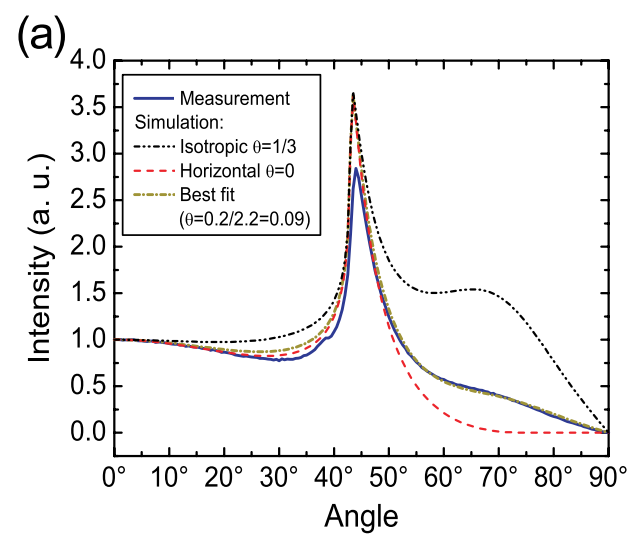

(b)

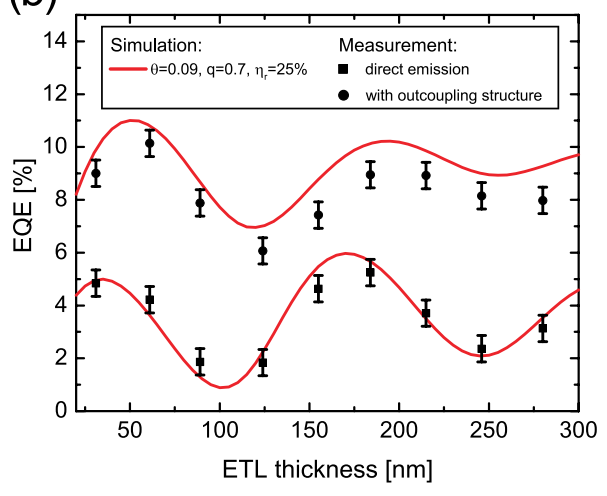

FIG. 2. (a) Determination of the anisotropy factor via p-polarized angular dependent photoluminescence measurement at a wavelength of 470 nm for a device without cathode and an ETL thickness of $67 \mathrm{~nm}$ (blue solid line: measured behavior; black dashed line: isotropic emitter orientation; red dashed line: horizontal emitter orientation; green dashed line: best fit). The best fit of the measured data is achieved with an emitter orientation of $\theta=0.2 / 2.2=0.09$. (b) Measured EQE values at a current density of $2 \mathrm{~mA} / \mathrm{cm}^{2}$. The lower solid line represents the best fit for the direct emission for $\eta_{\mathrm{r}}=25 \%$ and the mainly horizontal emitter orientation $(\theta=0.09$; determined from (a)), yielding an RQE of 0.7. The upper line illustrates the simulation for the extracted substrate modes as a function of the ETL thickness using the results of the fit of the direct emission. 
0.7 for a fixed $\eta_{\mathrm{r}}=25 \%$. Nevertheless, the simulation of the substrate modes is still not satisfactory and an additional mechanism has to be considered.

Thus, a possible enhancement of the radiative exciton fraction for fluorescent emitting dyes was investigated by electrically and optically pumped time-resolved measurements as shown in Fig. 3. All time-resolved spectra have been detected with a streak camera system (Hamamatsu C5680) combined with a spectrograph (Princeton Instruments Acton SpectraPro 2300i) and the excitation signal was synchronized with the streak camera system by a multichannel delay generator (Stanford Research Systems DG535). The electrical excitation was performed by an arbitrary waveform generator (Tabor Electronics WW2571A) with variable pulse length and repetition rate. Figure 3(a) illustrates a measured streak camera image for excitation with a square wave voltage of $4 \mathrm{~V}$ amplitude and $20 \mu \mathrm{s}$ length to achieve steady state conditions. Delayed fluorescence with the same spectrum as during the electrical pulse is visible. For clarity, Fig. 3(b) shows the time-resolved electroluminescence intensity decay integrated over all emitted wavelengths extracted from the streak camera image together with the simulated RC-time of the device.

The clear occurrence of a delayed component indicates that TTA and/or TADF are playing a major role for light emission. In order to clarify which of these two effects is the dominant one, time-resolved photoluminescence measurements have been performed by excitation with a short nitrogen laser pulse (Laser Technik Berlin MNL 200). The streak camera image, using an electronic gate function to cut off the strong prompt signal of the fluorescence, is illustrated in Fig. 3(c), again showing a strong delayed component with the same spectrum as for electrical excitation. It should be noted that the relative intensity of the delayed component under electrical operation is much higher than the fraction in the photoluminescence experiment, if the prompt fluorescence is not suppressed by the gate function of the streak camera system (see supplementary information for details ${ }^{29}$ ). This gives a first hint that the underlying effect might be TADF and not TTA. To further support this assumption, temperature dependent time-resolved photoluminescence measurements have been performed in a cryostat from $300 \mathrm{~K}$ down to $100 \mathrm{~K}$. Figure 3(d) illustrates the temperature dependent behavior of the delayed fluorescence under optical excitation. The strong decrease in the intensity of the delayed component accompanied by an increase in the emission lifetime (determined by mono-exponential fits) for falling temperature, indicates that TADF might be the dominant mechanism. This process requires a thermal activation to induce an efficient upconversion from the triplet to the singlet state of the dye and becomes more and more unlikely for decreasing temperature due to the decreasing Boltzmann factor. Nevertheless, TTA can be influenced by the temperature decrease, too, in terms of suppressing the diffusion of triplet excitons and therewith decreasing probability of bi-molecular processes. However, the absence of a red-shifted phosphorescence signal, which would be expected as a consequence of negligible triplet diffusion, indicates that the singlet-triplet splitting should be very small, which in turn favors TADF. All together, there is strong evidence that the dominant mechanism behind the delayed fluorescence is TADF, but an explicit proof would need intensity dependent measurements, which are beyond the scope of this article. Furthermore, knowing the exact mechanism is not required for the efficiency analysis presented here.

The appearance of a strong delayed fluorescence signal under electrical operation (regardless if it is caused by TADF or TTA) is a clear indicator for an enhanced radiative exciton fraction $\eta_{\mathrm{r}}$ beyond the spin-statistical limit. Thus, an independent method for the determination of $\eta_{\mathrm{r}}$ is required. For this purpose, we investigated the variations of the excited states lifetime of the prompt fluorescence as a function of the ETL thickness. Due to the very short excited
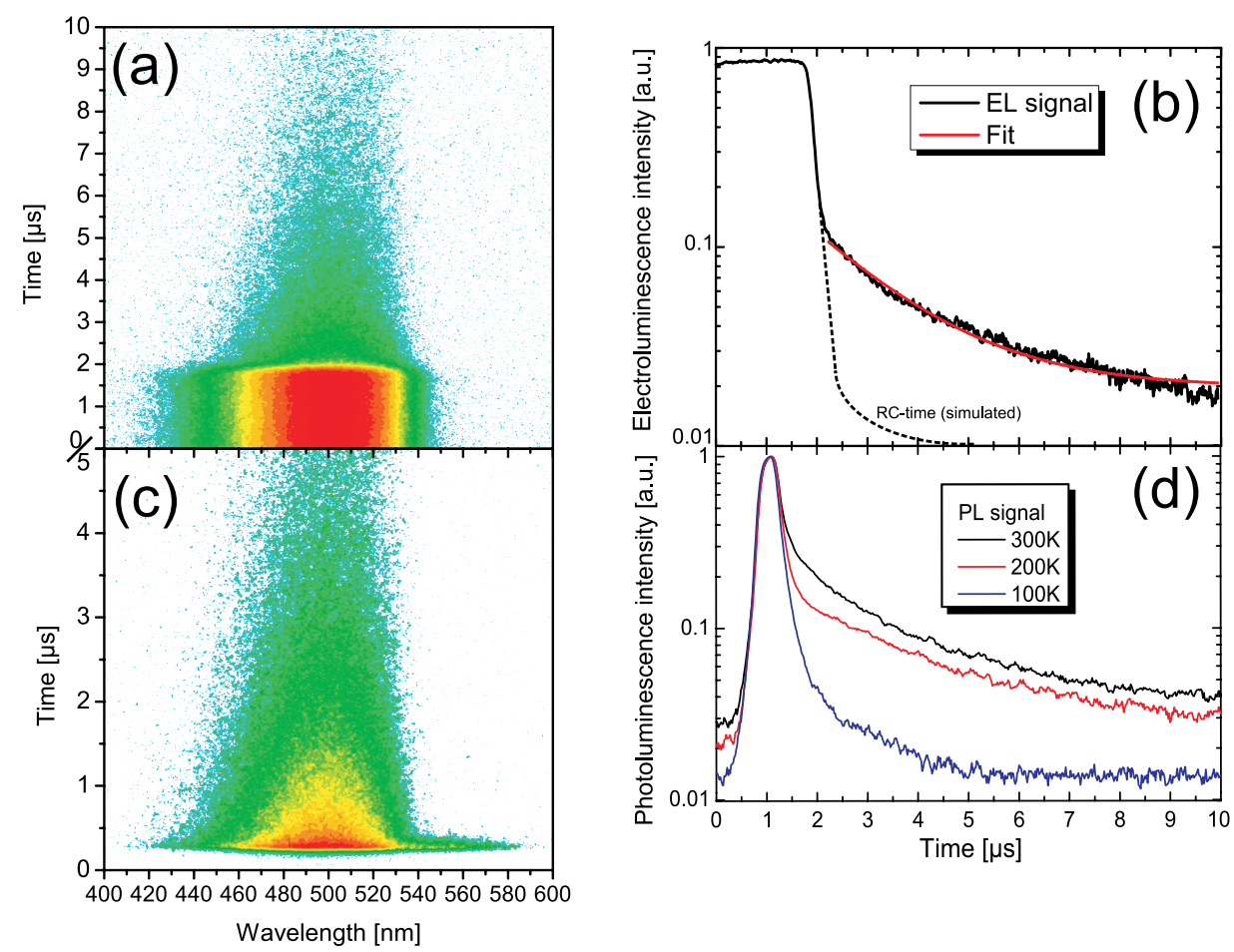

FIG. 3. (a) Time- and wavelengthdependent pulsed electroluminescence measurement of a device exhibiting an ETL thickness of $184 \mathrm{~nm}$. (b) Decay of the intensity integrated over the emission wavelength of (a). The initial fast drop of the electroluminescence is followed by a delayed long-living component with a lifetime of several $\mu$ s (mono-exponential fit: red line; simulated RC-time of the device: dashed line). (c) Time- and wavelengthdependent pulsed photoluminescence measurement of the same device. (d) Temperature dependent behavior of the delayed photoluminescence signal. The intensity of the delayed component decreases while the lifetime increases with falling temperature. Most of the intensity of the prompt fluorescence in (c) and (d) was cut off by the gate function of the used streak camera system to avoid saturation of the detector. 
states lifetimes of a few nanoseconds only, a laser diode (Hamamatsu PLP10-038) with a pulse duration of 80 ps was used for these investigations. The measured data points are plotted in Fig. 4 together with a fit based on optical simulations, ${ }^{22,24}$ including the prevailing horizontally oriented transition dipole moments of the emitting molecules and with the intrinsic radiative quantum efficiency $q$ and the intrinsic excited states lifetime $\tau_{0}$ of the emitting species as free parameters. The best fit results in values of $q$ and $\tau_{0}$ of $0.50 \pm 0.04$ and $(3.75 \pm 0.03) \mathrm{ns}$, respectively. Note, that this method is independent of the amount of light that is coupled out of the device and of delayed fluorescent components being caused by TADF and/or TTA, because the intersystem crossing rate should not be affected by the cavity environment due to its non-radiative character. The reason is that this rate only depends on the mixing of singlet and triplet states by the spin-orbit coupling operator and the energy separation between the mixing states. ${ }^{34,35}$

With this information, it is now possible to refine the efficiency analysis in a proper way and a determination of the lower limit of the enhanced radiative exciton fraction is possible. Figure 5 again illustrates the EQE measurements of the devices with varying ETL thickness together with simulations based on the above discussed different assumptions: (i) isotropic emitter orientation with $\eta_{\mathrm{r}}=25 \%$, (ii) mainly horizontal emitter orientation but still $\eta_{\mathrm{r}}=25 \%$, and (iii) mainly horizontal emitter orientation with $\eta_{\mathrm{r}}>25 \%$. While the direct emission can be described in a more or less acceptable way by all three assumptions, the situation is different for the extracted substrate modes of the devices. It is apparent that simulations with $\eta_{\mathrm{r}}=25 \%$ and the respective values for $q$ and $\theta$ taken from direct emission overestimate the contribution of substrate modes. A consistent description of both contributions can only be achieved, if an enhanced radiative exciton fraction due to triplet-to-singlet up-conversion (which is, by the way, not affected by variations of the cavity length) is taken into account. Fitting the measured data with $q$ and $\eta_{\mathrm{r}}$ as free parameters results in values of $0.45 \pm 0.05$ and $36 \pm 3 \%$, respectively. The first value is in excellent agreement with the RQE of 0.50 determined via the prompt

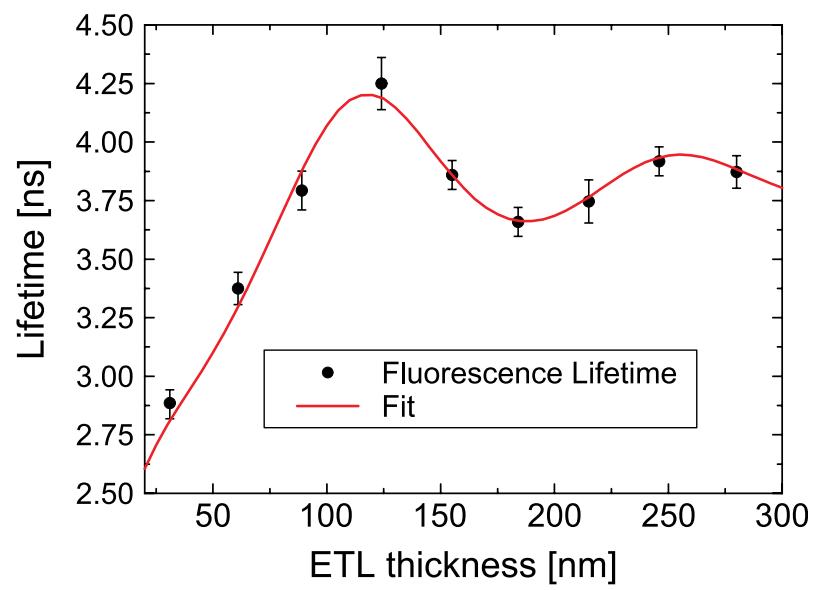

FIG. 4. Determination of the RQE of the emitting system via analysis of the excited states lifetimes of the prompt fluorescence of the devices as a function of the ETL thickness. The solid line is the best fit of the data points, including the mainly horizontal emitter orientation and results in $q=$ $0.50 \pm 0.04$ and an intrinsic excited states lifetime of $\tau_{0}=(3.75 \pm 0.03) \mathrm{ns}$.

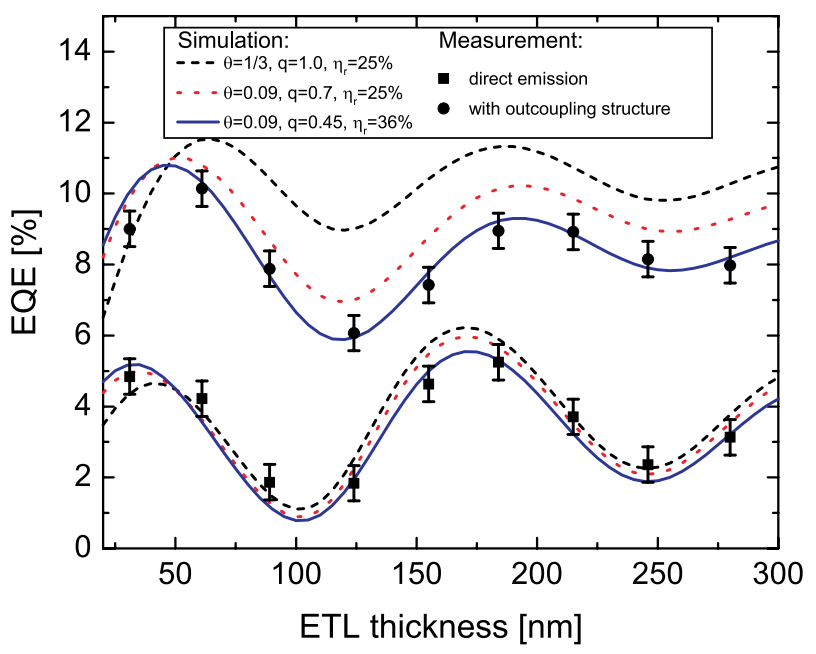

FIG. 5. Determination of the RQE of the emitting system via EQE measurements with (dots) and without (squares) macroextractor at a current density of $2 \mathrm{~mA} / \mathrm{cm}^{2}$. Solid lines represent simulations with three different assumptions: (i) Black dashed line: isotropic emitter orientation $(\theta=1 / 3)$ with a classical radiative exciton fraction $\left(\eta_{\mathrm{r}}\right)$ of $25 \%$ and an ideal radiative quantum efficiency $(q)$ of unity; (ii) red dotted line: mainly horizontal emitter orientation $\theta=0.09, \eta_{\mathrm{r}}=25 \%$, and $q=0.7$; (iii) blue solid line: $\theta=0.09$, $\eta_{\mathrm{r}}=36 \%$, and $q=0.45$.

fluorescence lifetimes (see Fig. 4). Strictly speaking, the obtained value for $\eta_{\mathrm{r}}$ should be understood as a lower limit, because the charge carrier balance was set to unity for the simulations and could in reality be slightly lower.

This clearly demonstrates the importance of including the substrate modes of OLEDs for a consistent efficiency analysis. Ignoring these effects for fluorescent dyes would lead to a huge overestimation of the RQE of the emitting system by up to a factor of about two.

In conclusion, we have presented a method to achieve a comprehensive efficiency analysis, which takes into account possible deviations from randomness of the emitter orientation and an enhanced radiative exciton fraction of the emitting system without further assumptions, e.g., about the triplet conversion mechanism. Moreover, this method is very general as it is applicable to any kind of OLED stack and no detailed information about the used materials are required. For the fluorescent system under investigation the efficiency is boosted by two effects. First, due to a horizontal alignment of the transition dipole moments of the emitting molecules, the outcoupling factor is enhanced by a factor of 1.3. Second, the lower limit for the radiative exciton fraction was determined to $36 \%$, resulting in an additional efficiency increase by a factor of 1.44 . As a consequence of the combination of both effects, the EQE almost doubles and values up to $5 \%$ are achieved for direct emission in spite of a comparatively low $q$ value of $45 \%$ only.

We acknowledge financial support by the German Federal Ministry of Education and Research (BMBF) under the Contract Nos. 13N10474 and 13N12240, namely, projects "TOPAS 2012" and "OLYMP," respectively. We also acknowledge financial support by the Deutsche Forschungsgemeinschaft (Contract No. BR 1728/13-1) as well as by Bayerische Forschungsstiftung (C. Mayr) and the Elite Network of Bavaria (J. Frischeisen). 
${ }^{1}$ Commercial white OLEDs are offered since 2010, e.g., by Osram and Philips.

${ }^{2}$ K. S. Yook and J. Y. Lee, Adv. Mater. 24, 3169 (2012).

${ }^{3}$ Y. Luo and H. Aziz, Adv. Funct. Mater. 20, 1285 (2010).

${ }^{4}$ D. Yokoyama, Y. Park, B. Kim, S. Kim, Y.-J. Pu, J. Kido, and J. Park, Appl. Phys. Lett. 99, 123303 (2011).

${ }^{5}$ Y.-J. Pu, G. Nakata, F. Satoh, H. Sasabe, D. Yokoyama, and J. Kido, Adv. Mater. 24, 1765 (2012).

${ }^{6}$ H. Sternlicht, G. C. Nieman, and G. W. Robinson, J. Chem. Phys. 38, 1326 (1963).

${ }^{7}$ A. Endo, K. Sato, K. Yoshimura, T. Kai, A. Kawada, H. Miyazaki, and C. Adachi, Appl. Phys. Lett. 98, 083302 (2011).

${ }^{8}$ K. Goushi, K. Yoshida, K. Sato, and C. Adachi, Nature Photon. 6, 253 (2012).

${ }^{9}$ T. Nakagawa, S.-Y. Ku, K.-T. Wong, and C. Adachi, Chem. Commun. 48, 9580 (2012).

${ }^{10}$ T. Tsutsui, E. Aminaka, C. P. Lin, and D. U. Kim, Philos. Trans. Soc. A 355, 801 (1997).

${ }^{11}$ M. Pfeiffer, K. Leo, X. Zhou, J. S. Huang, M. Hofmann, A. Werner, and J. Blochwitz-Nimoth, Org. Electron. 4, 89 (2003).

${ }^{12}$ C. Adachi, M. A. Baldo, M. E. Thompson, and S. R. Forrest, J. Appl. Phys. 90, 5048 (2001).

${ }^{13}$ M. A. Baldo, D. F. O'Brien, Y. You, A. Shoustikov, S. Sibley, M. E. Thompson, and S. R. Forrest, Nature 395, 151 (1998).

${ }^{14}$ J. S. Wilson, A. S. Dhoot, A. J. A. B. Seeley, M. S. Khan, A. Kohler, and R. H. Friend, Nature 413, 828 (2001).

${ }^{15}$ Y. Sun, N. C. Giebink, H. Kanno, B. Ma, M. E. Thompson, and S. R. Forrest, Nature 440, 908 (2006).

${ }^{16}$ M. A. Baldo, D. F. O'Brien, M. E. Thompson, and S. R. Forrest, Phys. Rev. B 60, 14422 (1999).

${ }^{17}$ M. Segal, M. A. Baldo, R. J. Holmes, S. R. Forrest, and Z. G. Soos, Phys. Rev. B 68, 075211 (2003).

${ }^{18}$ S. Nowy, B. C. Krummacher, J. Frischeisen, N. A. Reinke, and W. Brütting, J. Appl. Phys. 104, 123109 (2008).
${ }^{19}$ H. Becker, S. E. Burns, and R. H. Friend, Phys. Rev. B 56, 1893 (1997).

${ }^{20}$ B. C. Krummacher, S. Nowy, J. Frischeisen, M. Klein, and W. Brütting, Org. Electron. 10, 478 (2009).

${ }^{21}$ M. Flämmich, J. Frischeisen, D. S. Setz, D. Michaelis, B. C. Krummacher, T. D. Schmidt, W. Brütting, and N. Danz, Org. Electron. 12, 1663 (2011).

${ }^{22}$ T. D. Schmidt, D. S. Setz, M. Flämmich, J. Frischeisen, D. Michaelis, B. C. Krummacher, N. Danz, and W. Brütting, Appl. Phys. Lett. 99, 163302 (2011).

${ }^{23}$ T. D. Schmidt, M. Flämmich, B. J. Scholz, D. Michaelis, C. Mayr, N. Danz, and W. Brütting, Proc. SPIE 8435, 843513 (2012).

${ }^{24}$ D. S. Setz, T. D. Schmidt, M. Flämmich, S. Nowy, J. Frischeisen, B. C. Krummacher, T. Dobbertin, K. Heuser, D. Michaelis, N. Danz, W. Brütting, and A. Winnacker, J. Photon. Energy 1, 011006 (2011).

${ }^{25}$ N. Danz, M. Flämmich, D. S. Setz, B. C. Krummacher, D. Michaelis, and T. Dobbertin, Opt. Lett. 37, 4134 (2012).

${ }^{26}$ N. C. Giebink, B. W. D. Andrade, M. S. Weaver, P. B. Mackenzie, J. J. Brown, M. E. Thompson, and S. R. Forrest, J. Appl. Phys. 103, 044509 (2008).

${ }^{27}$ N. C. Giebink and S. R. Forrest, Phys. Rev. B 77, 235215 (2008).

${ }^{28}$ S. Reineke, K. Walzer, and K. Leo, Phys. Rev. B 75, 125328 (2007).

${ }^{29}$ See supplementary material at http://dx.doi.org/10.1063/1.4819388 for the investigation of the efficiency roll-off of the devices under investigation and for the illustration of the prompt fluorescence of the emitting species.

${ }^{30}$ J. Frischeisen, D. Yokoyama, A. Endo, C. Adachi, and W. Brütting, Org. Electron. 12, 809 (2011).

${ }^{31}$ D. Yokoyama, J. Mater. Chem. 21, 19187 (2011).

${ }^{32}$ J. Frischeisen, D. Yokoyama, C. Adachi, and W. Brütting, Appl. Phys. Lett. 96, 073302 (2010).

${ }^{33}$ M. Flämmich, M. C. Gather, N. Danz, D. Michaelis, A. H. Bräuer, K. Meerholz, and A. Tünnermann, Org. Electron. 11, 1039 (2010).

${ }^{34}$ A. Köhler and H. Bässler, Mater. Sci. Eng., R. 66, 71 (2009).

${ }^{35}$ Highly Efficient OLEDs With Phosphorescent Materials, edited by $\mathrm{H}$. Yersin (Wiley-VCH, Berlin, 2007). 